\title{
Combined Effects of Glucocorticoid and Noradrenergic Activity on Loss Aversion
}

\author{
Zsofia Margittai', Gideon Nave², Marijn Van Wingerden', Alfons Schnitzler ${ }^{3}$, Lars Schwabe ${ }^{4}$ and \\ Tobias Kalenscher*,I \\ 'Comparative Psychology, Heinrich Heine University, Düsseldorf, Germany; ${ }^{2}$ Department of Marketing, The Wharton School, University of \\ Pennsylvania, Philadelphia, PA, USA; ${ }^{3}$ Institute of Clinical Neuroscience and Medical Psychology, Heinrich Heine University, Düsseldorf, Germany; \\ ${ }^{4}$ Department of Cognitive Psychology, Institute of Psychology, University of Hamburg, Hamburg, Germany
}

\begin{abstract}
Loss aversion is a well-known behavioral regularity in financial decision making, describing humans' tendency to overweigh losses compared to gains of the same amount. Recent research indicates that stress and associated hormonal changes affect loss aversion, yet the underlying neuroendocrine mechanisms are still poorly understood. Here, we investigated the causal influence of two major stress neuromodulators, cortisol and noradrenaline, on loss aversion during financial decision making. In a double-blind, placebo-controlled between-subject design, we orally administered either the $\alpha$ 2-adrenergic antagonist yohimbine (increasing noradrenergic stimulation), hydrocortisone, both substances, or a placebo to healthy young men. We tested the treatments' influence on a financial decision-making task measuring loss aversion and risk attitude. We found that both drugs combined, relative to either drug by itself, reduced loss aversion in the absence of an effect on risk attitude or choice consistency. Our data suggest that concurrent glucocorticoid and noradrenergic activity prompts an alignment of reward- with loss-sensitivity, and thus diminishes loss aversion. Our results have implications for the understanding of the susceptibility to biases in decision making.

Neuropsychopharmacology (2018) 43, 334-34I; doi:I0.1038/npp.2017.75; published online 10 May 2017
\end{abstract}

\section{INTRODUCTION}

Most people would prefer to avoid losing 5 EUR than to win 5 EUR. This phenomenon is called loss aversion, a wellknown behavioral regularity in financial decision making, describing humans' tendency to overweigh losses compared to gains of the same amounts (Kahneman and Tversky, 1979). Accumulating evidence suggests that stress has an impact on loss aversion (Porcelli and Delgado, 2009; Putman et al, 2010; Takahashi et al, 2012; Pabst et al, 2013a; Chumbley et al, 2014; Sokol-Hessner et al, 2015), however, there is fundamental disagreement on the direction of the effects, and the underlying neuroendocrine mechanisms are largely unknown.

Organisms respond to acute stress with a rapid release of noradrenaline (NA) through the sympathetic nervous system and a slower release of glucocorticoids (mainly cortisol (CORT) in humans) as the end-product of the hypothalamic-pituitary-adrenal axis. The effects of CORT and NA on brain function shape cognition and behavior in a concerted, time-dependent fashion (Joels and Baram, 2009; Joëls et al, 2011; Schwabe et al, 2012), characterized by overlapping,

*Correspondence: $\operatorname{Dr} T$ Kalenscher, Heinrich Heine University, Comparative Psychology, Universitätsstrasse I, 40225 Düsseldorf, Germany, Tel: +49 21 | 81 | | |607, Fax: +49 21| 81। 1629,

E-mail: Tobias.Kalenscher@hhu.de

Received 4 January 2017; revised 21 March 2017; accepted 4 April 2017; accepted article preview onlinel4 April 2017 non-genomic cortisol and catecholaminergic action shortly after stress onset, followed by genomic cortisol effects that develop several hours later and can have opposite effects on brain function to those in the immediate aftermath of stress (Hermans et al, 2014).

In the present experiment, we contrast two competing hypotheses on how CORT and NA influence loss aversion. Recent findings on the effect of stress hormone action on cognition and decision-making suggest that stress prompts the upregulation of a salience network in the brain, including insula, amygdala, and other limbic regions, while simultaneously suppressing higher cognitive prefrontal control networks (Hermans et al, 2011, 2014; Schwabe et al, 2012; Margittai et al, 2016), resulting in hypervigilance to potential losses. Activation of the salience network is boosted by catecholamines, such as noradrenaline, whose effects are further enhanced when combined with glucocorticoids such as cortisol (van Stegeren et al, 2008, 2010; Hermans et al, 2011, 2014). Importantly, two key regions of the salience network, the amygdala (De Martino et al, 2010; Sokol-Hessner et al, 2013; Gelskov et al, 2015), and insula (Canessa et al, 2013; Markett et al, 2016), have been associated with loss aversion, likely by mediating attention to potential losses. On the basis of this presumption, the 'salience of losses' hypothesis postulates that combined action of the stress-neuromodulators NA and CORT should amplify loss aversion by boosting loss-related neural 
functioning, and thus enhancing the salience of potential losses.

By contrast, CORT and NA are also known to impact reward processing (Pruessner et al, 2003; Starcke and Brand, 2012). For example, Mather and Lighthall (2012) proposed that stress can increase the salience of rewards, and enhance learning about positive choice outcomes while impairing learning about negative outcomes. In line with this presumption, several studies have reported that acute stress leads to increased reward sensitivity (Sinha, 2008; van den Bos et al, 2009; Putman et al, 2010) and decreased loss sensitivity in risky decision making (Pabst et al, 2013a, 2013b). In a recent meta-analysis, Starcke and Brand (2016) reported that stress affects decisions made under risk and ambiguity, particularly in situations where reward seeking is disadvantageous. Importantly, the direction of the stress effect on reward and loss sensitivity seems to depend on the concomitance of the stress-neuromodulators CORT and NA. While isolated exogenous administration of CORT (ie, in the absence of elevated NA) has been shown to reduce reward sensitivity and neural activation in reward-related regions (Montoya et al, 2014; Kinner et al, 2016), concurrent CORT and NA action is associated with enhanced reward sensitivity and ventral striatal activation, particularly at higher levels of cortisol elevations (Oei et al, 2014), as well as reduced anxiety and vigilance to threat (Vasa et al, 2009). On the basis of these findings the 'alignment hypothesis' suggests that when CORT and NA act concurrently, CORT may offset NAinduced vigilance to threats by amplifying reward sensitivity, presumably by stimulating dopaminergic release in the midbrain mesolimbic reward pathway, in particular the nucleus accumbens (Piazza and Moal, 1997; Marinelli and Piazza, 2002; Oei et al, 2014). Hence, the alignment hypothesis predicts that combined CORT and NA action results in an alignment of reward- with threat-susceptibility and, thus, ultimately, diminished loss aversion.

No study to date has delineated whether stress neuromodulators increase the salience of expected losses, as conjectured by the salience-of-losses hypothesis, or prompt an alignment of reward- with loss-sensitivity, as hypothesized by the alignment hypothesis. Importantly, these two theories make opposing predictions regarding the effects of stress neuromodulators on loss aversion. While the salience-oflosses hypothesis predicts linear, additive effects of CORT and NA on loss sensitivity, resulting in increased loss aversion, the alignment hypothesis predicts that CORT and NA in concert diminish loss aversion relative to either neuromodulator alone. To contrast these theoretical predictions, we pharmacologically manipulated CORT and NA in a double-blind, placebo-controlled experimental design and probed their combined and isolated causal effects on loss aversion.

\section{MATERIALS AND METHODS}

\section{Participants}

Ninety-two healthy, male participants took part in the experiment after prior eligibility screening. Individuals who reported the use of medication, psychiatric or medical treatment, acute or chronic illness, heavy smoking, drinking, regular drug use, or enrollment in Economics or Psychology study programs were excluded from participation. All participants were fluent German speakers, gave their informed consent and received financial compensation for their participation. The study was carried out in line with the Declaration of Helsinki and was approved by the medical ethics committee of the University Hospital Düsseldorf. Participants were asked to refrain from sexual activities $24 \mathrm{~h}$ before participation, not to smoke, eat, and drink anything other than water, and to avoid

Table I Demographic and Trait Control Measures

\begin{tabular}{|c|c|c|c|c|c|c|c|c|c|c|}
\hline & Placebo & Yohimbine & Cortisol & YohCort & F (YOH) & $p$ & F (CORT) & $p$ & F (YOHCORT) & $p$ \\
\hline & $M(\mathrm{SD})$ & $M(\mathrm{SD})$ & $M(\mathrm{SD})$ & $M(\mathrm{SD})$ & & & & & & \\
\hline BMI & $22.96(2.50)$ & $22.62(2.10)$ & $22.90(2.13)$ & $22.96(2.40)$ & 0.08 & 0.773 & 0.08 & 0.774 & 0.17 & 0.680 \\
\hline Baseline cortisol & | 5.57 (| 3.90$)$ & $\mid 4.42(9.3 \mid)$ & $|2.5|(6.39)$ & $|4.4|(8.65)$ & 0.03 & 0.856 & 0.54 & 0.466 & 0.53 & 0.469 \\
\hline STAI & $41.41(11.29)$ & $37.10(6.04)$ & $36.17(9.84)$ & $38.00(9.25)$ & 0.39 & 0.536 & 1.19 & 0.279 & 2.37 & 0.127 \\
\hline Empathy (SPF) & $41.55(6.13)$ & $42.10(7.46)$ & $41.54(4.27)$ & $41.39(6.78)$ & 0.02 & 0.879 & 0.07 & 0.788 & 0.07 & 0.790 \\
\hline BIS-I5 & $32.41(7.93)$ & $31.60(4.75)$ & $31.92(6.84)$ & $32.30(5.64)$ & 0.02 & 0.878 & 0.01 & 0.938 & 0.19 & 0.663 \\
\hline BIS & $20.00(4.42)$ & I $8.45(3.72)$ & I $8.00(3.19)$ & $19.17(3.46)$ & 0.06 & 0.812 & 0.66 & 0.421 & 2.98 & 0.088 \\
\hline BAS & $41.32(4.02)$ & $42.55(4.01)$ & $43.00(3.84)$ & $41.17(4.64)$ & 0.11 & 0.736 & 0.03 & 0.862 & 3.02 & 0.086 \\
\hline
\end{tabular}

Abbreviations: BAS, Behavioral Approach Scale; BIS-I5, Short version of the Barratt Impulsiveness Scale; BIS, Behavioral Inhibition Scale; BMI, body mass index; F (CORT), main effect of hydrocortisone; F (YOHCORT), yohimbine $\times$ hydrocortisone interaction; F (YOH), main effect of yohimbine; sAA, salivary alpha amylase; SDS- I7, Social Desirability Scale; SPF, Saarbrücker Persönlichkeitsfragebogen (German version of the Interpersonal Reactivity Index); STAI, State Trait Anxiety Inventory; VAS, visual analog scale. 
exercising $2 \mathrm{~h}$ before the experiment. These criteria were similar to those employed in prior studies, (eg Vinkers et al, 2013). Two participants were unable to complete the experimental tasks due to technical failure and were thus excluded from all analyses. One further participant was excluded because he exclusively selected the gamble option on all trials, irrespective of the payoff and risk contingencies. We used an exclusively male sample in order to avoid differential HPA-axis activation caused by the intake of oral contraceptives and variations in menstrual cycle (Kirschbaum et al, 1999). See Table 1 for demographic measures.

\section{Trait Measures}

In order to control for potential trait confounds between treatment groups, participants completed a number of online questionnaires several days before testing. We recorded trait anxiety (State Trait Anxiety Inventory-STAI, Spielberger et al, 1983), impulsivity (Barratt Impulsiveness ScaleBIS-15; Meule et al, 2011), reward and punishment sensitivity (BIS/BAS scale; Carver and White, 1994), social desirability (SDS-17; Ströber, 2001), general willingness to take risks, chronotype (short version of the MorningnessEveningness Questionnaire; Randler, 2013), and empathy (Saarbrücker Persönlichkeitsfragebogen, Paulus, 2007, which is a German version of the Interpersonal Reactivity Index, Davis, 1980). See Table 1 for statistics.

\section{Pharmacological Manipulation, Physiological and Subjective Stress Measures}

In a double-blind, placebo-controlled experimental design, participants were randomly assigned to one of four experimental groups: (A) placebo $(N=24)$, (B) placebo + yohimbine (20 mg, Cheplapharm, $N=21$ ), (C) placebo + hydrocortisone $(20 \mathrm{mg}$, Jenapharm, $N=24)$, (D) yohimbine + hydrocortisone $(20 \mathrm{mg}$ each, $N=23)$. Dosage was in line with prior studies (Schwabe et al, 2010; van Stegeren et al, 2010). To assess the effectiveness of the treatment, saliva samples were collected at baseline and $+30,+45$, and +75 min after pill intake using Salivette devices (Sarstedt, Germany) and subsequently analyzed for cortisol and alphaamylase (sAA, a marker of noradrenergic activity) concentrations. Samples were frozen and stored at $-20^{\circ} \mathrm{C}$ until analysis, and they were analyzed as reported by Rohleder et al (2004). In total, 15 of the 460 saliva samples used for analysis were compromised, missing data were excluded from the analysis. Subjective stress ratings were assessed using visual analog scales (VAS, $100 \mathrm{~mm}$ scale) at the same time points as the saliva samples.

\section{Risk and Loss Aversion Task}

The experimental task (Wang et al, 2010) was similar to that used by Sokol-Hessner et al (2009), Chumbley et al (2014) and Sokol-Hessner et al (2015). Participants made 40 binary choices between receiving amount $(x)$ for sure and a lottery, where they had a 0.5 probability of either winning amount $(y)$ or losing amount $(z)$, Figure 1. The task used an adaptive design; thus, choice options were dynamically selected based on participants' prior answers, according to an informational criterion that optimized the estimation of individual

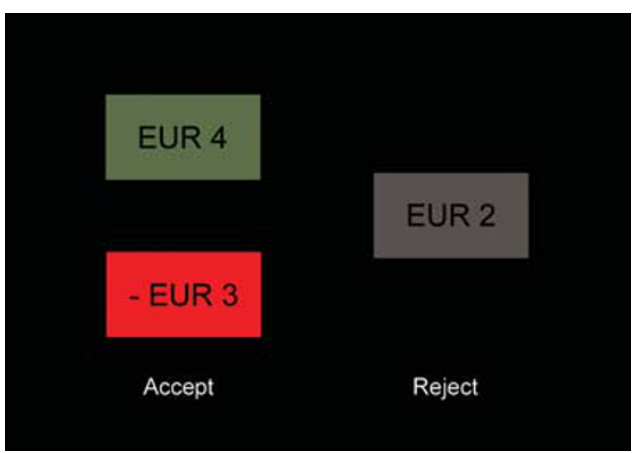

Figure I Example decision screen of the experimental task. Participants had to decide, whether they would like to gamble (in this example 50\% chance of winning 4 Euros and 50\% chance of losing 3 Euros), or choose the safe option (in this example, winning 2 Euros for sure).

parameters describing loss aversion $(\lambda)$, and risk aversion $(\rho)$. In line with Sokol-Hessner et al $(2009,2015)$, we used the prospect-theory (Kahneman and Tversky, 1979) inspired utility function $u(w+)=w^{\rho}$ to determine positive payoffs $(w+)$, and $u(w-)=-\lambda(w)^{\rho}$ to determine negative payoffs $(w-)$. Next, we fitted a softmax function (equation 1) to the data, such that the probability of choosing the risky lottery for a given utility function was:

$$
p(\text { lottery } \mid \rho, \lambda, \mu)=\frac{1}{1+e^{-\mu\left(\frac{1}{2} u(x)+\frac{1}{2} u(z)-u(y)\right)}}
$$

where the nuisance parameter $(\mu)$ assesses consistency in choice behavior. Note that $\lambda>1$ indicate loss aversion, $\lambda<1$ indicate loss seeking and $\lambda=1$ indicates loss neutrality. $\rho>1$ indicate risk aversion, $\rho<1$ indicate risk seeking and $\rho=1$ indicates risk neutrality. We log-transformed $\lambda$ values for all analyses, a common approach (Sokol-Hessner et al, 2015) because the distribution of $\lambda$ is positively skewed. The experiment was incentive compatible: upon completion of the session one of participants' choices was chosen at random, played, and paid out. Participants were aware of this before the commencement of the task.

\section{Procedure}

All experimental sessions took place in the afternoon between 14:00 and 18:00 hours in order to control for diurnal variations of cortisol levels. Upon arrival at the laboratory, participants were asked to give their informed consent and complete a number of baseline measures (Table 1). Thereafter participants consumed the drugs. After a waiting period of $45 \mathrm{~min}$ (Schwabe et al, 2010) participants were asked to start with the experimental tasks. The experiment included three separate tasks in a counterbalanced order: the present task and two unrelated tasks that were reported elsewhere (Margittai et al, 2016). The total duration of the three tasks was $\sim 20 \mathrm{~min}$.

\section{Analyses of Trait and Baseline Measures, Pharmacological Manipulation Check and Analysis of Loss- and Risk Aversion Parameters}

We analyzed trait and baseline measures using univariate analyses of variance (ANOVA) with the between-subject 
factors noradrenergic activation (yohimbine $v s$ placebo), and cortisol administration (hydrocortisone vs placebo). Analyses of the loss- and risk-aversion parameters were performed in a similar fashion.

As a confirmation of our pharmacological manipulation, we analyzed baseline-corrected changes in salivary cortisol and salivary alpha-amylase- and baseline-corrected changes in subjective feelings of stress using mixed ANOVAs (between-subject factor: noradrenergic activation (yohimbine vs placebo) and cortisol administration (hydrocortisone vs placebo), within subject factor: time point of measurement.

\section{RESULTS}

\section{Trait and Baseline Measures}

Our analyses showed no significant differences in baseline, demographic or trait measures between the groups (Table 1).

\section{Manipulation Check}

Salivary cortisol and alpha amylase. Salivary cortisol significantly increased in participants taking hydrocortisone; main effect of hydrocortisone on salivary cortisol: $\mathrm{F}_{1,78}=100.24, p<0.001, \eta_{\mathrm{p}}^{2}=0.56$; time point $\times$ hydrocortisone interaction $\mathrm{F}_{1.15,89.83}=12.44, p<0.001, \eta_{\mathrm{p}}^{2}=0.14$. There was no significant effect of yohimbine on salivary cortisol $\left(\mathrm{F}_{1,78}=0.60, p=0.440, \eta_{\mathrm{p}}^{2}=0.01\right)$ nor a cortisol $\times$ yohimbine interaction $\left(\mathrm{F}_{1,78}=0.42, p=0.520, \eta_{\mathrm{p}}^{2}=0.01\right)$, indicating that yohimbine did not alter cortisol levels, Figure 2a. Conversely, sAA increased significantly after taking yohimbine: $\mathrm{F}_{1,83}=16.84, p<0.001, \eta_{\mathrm{p}}^{2}=0.17$, time point $\times$ yohimbine interaction $\left(\mathrm{F}_{1.52,126.14}=4.68, p=0.018, \eta_{\mathrm{p}}^{2}=0.05\right)$, but not after taking hydrocortisone: $\left(\mathrm{F}_{1,83}=0.14, \quad p=0.710\right.$, $\left.\eta_{\mathrm{p}}^{2}=0.002\right)$. There was no hydrocortisone $\times$ yohimbine interaction on sAA levels $\left(\mathrm{F}_{1,83}=0.06, p=0.815\right.$, $\eta_{\mathrm{p}}^{2}=0.001$ ), indicating that hydrocortisone did not alter sAA levels, Figure $2 \mathrm{~b}$.

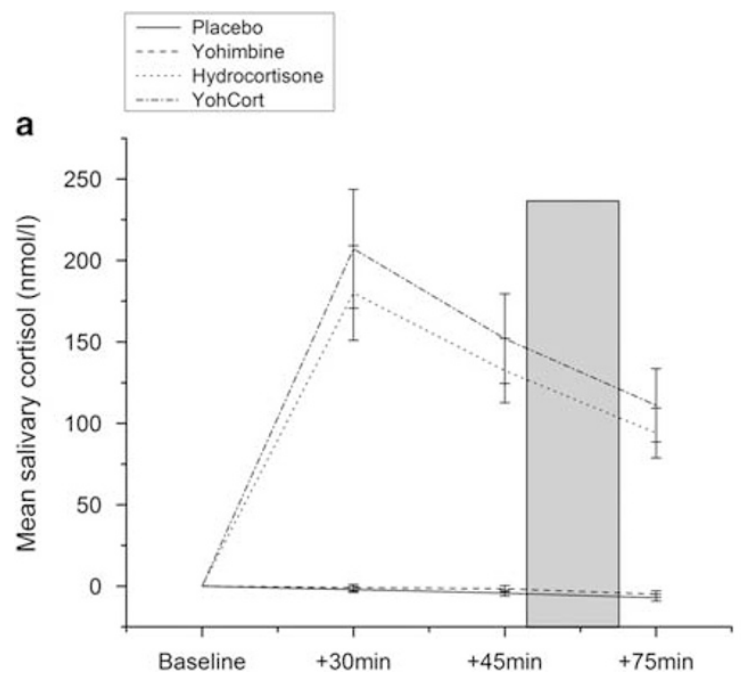

Figure 2 Baseline-corrected increases in salivary cortisol and alpha-amylase. (a) Salivary cortisol increased significantly $(p<0.00 \mathrm{I})$ over time after taking hydrocortisone in the hydrocortisone and yohimbine+cortisol (yohcort) groups. (b) Salivary alpha-amylase also increased significantly $(p<0.05)$ over time after taking yohimbine in the yohimbine and yohcort groups. The gray bars indicate the time of behavioral testing. Error bars indicate \pm I SEM.

\section{Subjective Stress Ratings}

Baseline-corrected changes in subjective feelings of stress increased in participants who received yohimbine (main effect of yohimbine: $\left.\mathrm{F}_{1,85}=5.75, p=0.019, \eta_{\mathrm{p}}^{2}=0.06\right)$, in line with prior research (eg, Elman et al, 2012), but not in those who received cortisol (main effect of hydrocortisone: $\left.\mathrm{F}_{1,85}=0.27, p=0.61, \eta_{\mathrm{p}}^{2}=0.003\right)$. There was no interaction between the two substances on subjective feelings of stress, nor a time point $\times$ hydrocortisone interaction, nor a three way interaction between yohimbine, hydrocortisone, and time point of testing $(p>0.141)$. This is in line with existing results indicating that cortisol administration does not usually result in changes in subjective affect or mood (Schwabe et al, 2010, 2012).

\section{Hydrocortisone and Yohimbine Jointly Reduce Loss Aversion but do not Affect Risk Attitude}

To assess the influence of cortisol and noradrenaline on loss aversion and risk attitude, we analyzed individual loss aversion and risk attitude parameters using a $2 \times 2$ betweensubjects ANOVA with the factors noradrenergic activation (yohimbine vs placebo) and cortisol administration (hydrocortisone $v s$ placebo). This analysis revealed a significant interaction effect of noradrenergic and cortisol activation on the loss aversion parameter $\log (\lambda),\left(\mathrm{F}_{1,85}=6.37, p=0.013\right.$, $\eta_{\mathrm{p}}^{2}=0.07$, Figure $3 \mathrm{~b}$ ). Breaking down this interaction effect, an independent samples $t$-test revealed that hydrocortisone significantly reduced $\log (\lambda)$, but only when accompanied by yohimbine intake $(t(45)=2.46, p=0.018, d=0.73$; Bonferroni-Holm corrected), and yohimbine decreased $\log (\lambda)$ depending on hydrocortisone availability $(t(41)=2.35, p=0.023$, $d=0.76$; Bonferroni-Holm corrected, Figure $3 \mathrm{~b}$ ). Finally, loss aversion in individuals who received both drugs was significantly lower compared to all other participants risk aversion parameter $\rho$ or the consistency parameter $\mu$ as

\section{b}

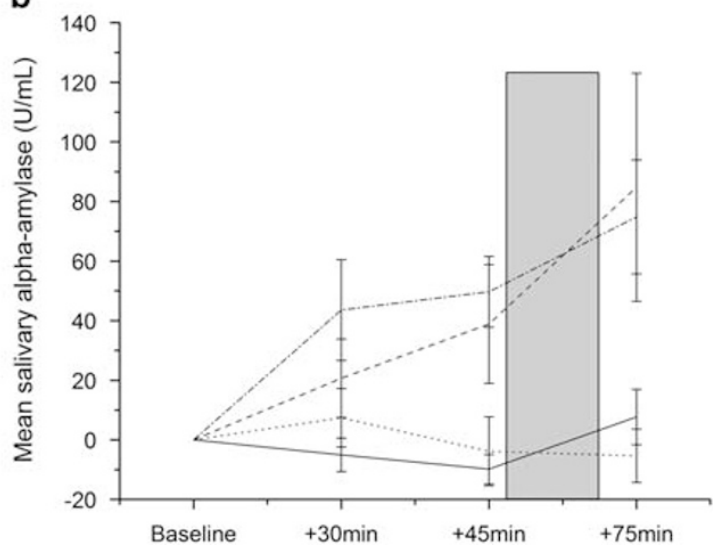
$(t(87)=2.41, p=0.018, d=0.34)$. A similar analysis with the 
No Hydrocortisone

Hydrocortisone

a

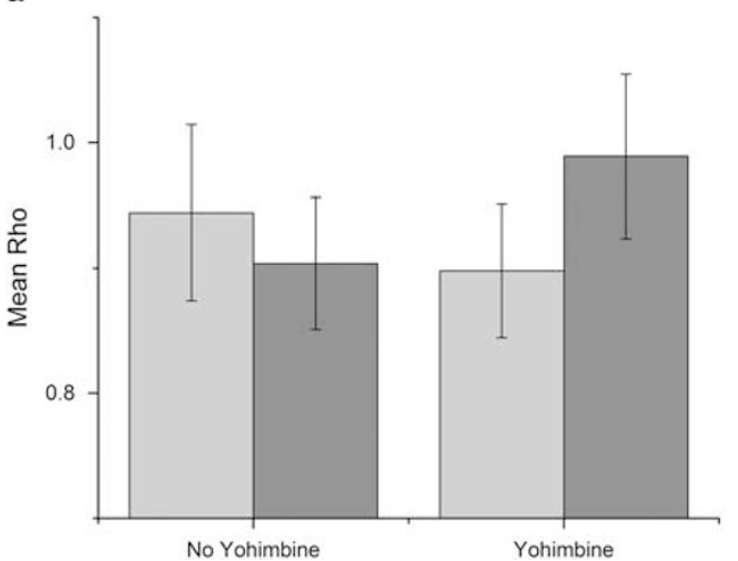

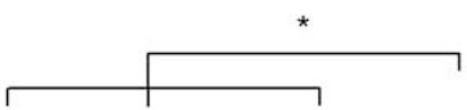

b

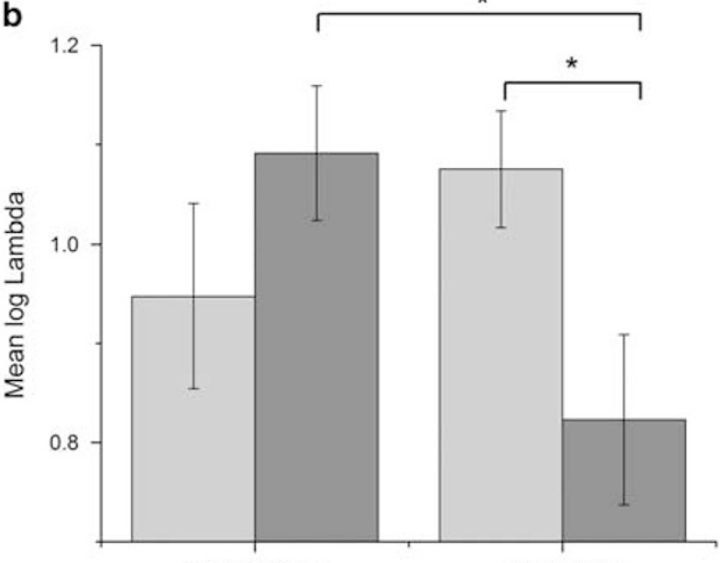

No Yohimbine

Yohimbine

Figure 3 (a) Risk aversion parameters were not affected by the pharmacological manipulation. (b) The effects of hydrocortisone and yohimbine on loss aversion. Individuals who received yohimbine and hydrocortisone had reduced loss aversion compared to those who received either substance alone. Loss aversion in the yohimbine+hydrocortisone group was also lower compared with all other participants. Significant effects $(p<0.05)$ are indicated by an asterisk. Error bars indicate \pm I SEM.

the dependent variables revealed no significant main or interaction effects of drugs (all $p>0.286$, Figure $3 a$ ).

\section{Treatment Expectancy}

Following the experimental tasks, we asked participants to indicate whether they believed to have received placebo or an active substance during the experiment. The number of participants who believed to have been given placebo vs active substances did not significantly differ between the four treatment conditions $\left(\chi^{2}(3, N=89)=6.63, p=0.085\right)$. To further rule out expectancy effects, a point biserial correlation revealed no significant relationship between belief about treatment (placebo $v s$ active substance) and the loss aversion parameter $(r=-0.11, p=0.298)$. Thus, belief about treatment is unlikely to have interfered with the results.

\section{DISCUSSION}

Stress is known to alter loss aversion (Porcelli and Delgado, 2009; Takahashi et al, 2012; Pabst et al, 2013a, 2013b; Chumbley et al, 2014), but the underlying neuroendocrine mechanisms have remained elusive so far. Here, we examined the causal effects of the exogenous manipulation of two stress neuromodulators, CORT, and NA, on loss aversion. We found that cortisol combined with noradrenergic stimulation diminished loss aversion relative to the action of either drug alone. By contrast, we found no drug effects on risk attitude or choice consistency measured in the same financial decision-making paradigm.

Our study provides evidence for a putative neuroendocrine mechanism underlying loss aversion. Our results are in disagreement with the predictions of the salience-of-losses theory according to which NA and CORT have additive effects on the salience of anticipated punishment and losses
(Gullo and Stieger, 2011), and thus loss aversion. By contrast, our data support the alignment hypothesis according to which the combined action of NA and CORT decreases loss aversion, presumably through the alignment of reward- to punishment-susceptibility associated with the upregulation of reward-sensitivity (Piazza and Moal, 1997; Marinelli and Piazza, 2002; Oei et al, 2014). This conclusion is in line with a recent neuroimaging study showing that elevated cortisol was associated with increased activity in reward-processing regions such as the nucleus accumbens after behaviorally induced stress (Oei et al, 2014).

Through the systematic manipulation of both NA and CORT, which allowed us to disentangle the isolated and combined effects of these two stress neuromodulators on loss aversion, we were able to demonstrate that NA and CORT in concert, but not in isolation, prompt an alignment of rewardand punishment-sensitivity. Thus, our results contribute to resolving contradictions in previous results regarding the direction of the stress effect on loss aversion, and its precise neuroendocrine foundation, and hopes to pave the way towards a unified theory on the neuroendocrine mechanisms underlying decision making under risk.

While some studies (Putman et al, 2010; Takahashi et al, 2012; Chumbley et al, 2014; Sokol-Hessner et al, 2015) reported stress or stress neuromodulator action to be negatively correlated with loss aversion, others found a positive relationship (Rogers et al, 2004; Porcelli and Delgado, 2009; Sokol-Hessner et al, 2009). In addition, one study by Sokol-Hessner et al (2016) found no effects of the cold-pressor task on loss aversion. These studies either correlated arousal-an indirect manifestation of sympathetic activity-with loss aversion (Sokol-Hessner et al, 2009, 2013), reduced NA-activity via exogenous administration of beta-blockers (Rogers et al, 2004; Sokol-Hessner et al, 2015), correlated endogenous measures of long term CORT exposure (extracted from hair cells) with loss aversion 
(Chumbley et al, 2014), or administered only CORT (Putman et al, 2010).

Overall, while all of the aforementioned studies focused on either CORT or NA alone, none of them controlled for the respective other stress neuromodulator, or systematically explored the interplay of NA and CORT on loss aversion. Consequently, given the neurohormonal interplay reported here, the influences of one hormone on loss aversion was likely modulated by the uncontrolled action of the other hormone, resulting in heterogeneous findings. In addition, procedural differences are also likely to account for some of the disparity between existing findings. More specifically, while some studies used a behavioral stress induction, eg, Porcelli and Delgado, 2009; Pabst et al, 2013a, 2013b; SokolHessner et al, 2016, our primary focus was a pharmacological manipulation to investigate the causal effects of the two main stress mediators on loss aversion. The effects are thus not directly comparable, as pharmacological manipulations, such as the one used in the present experiment, have some differences from naturally occurring stress. For instance, they tend to result in supraphysiological levels of the neuromodulators compared to natural stress (Lupien et al, 1999), and do not bring about the affective response to threat in the same way that naturally occurring stress usually does. This point is important to keep in mind, as it has been reported in prior research that low elevations in cortisol may have a different, even opposite, effect on reward-sensitivity (Oei et al, 2014) than higher elevations. However, an advantage of a pharmacological design is that allows for precise, causal conclusions about the effects of cortisol and noradrenaline on behavior.

In line with the above, a review published by Porcelli and Delgado, 2017 also emphasizes the wide array of methodological and theoretical differences present in existing research that pose a challenge for the comparison of findings. In addition to not controlling systematically for both neuromodulators, the different stress induction procedures are likely to result in different elevations in neuromodulator levels, and the timing of the behavioral tasks in relation to stress onset may also explain some of the disparity between findings (Pabst et al, 2013b; Starcke and Brand, 2016).

Whereas our findings showed a striking interactive effect of our cortisol and noradrenergic action on loss aversion, the stress neuromodulator manipulation did not affect risk aversion $(\rho)$. This is consistent with other reports (Chumbley et al, 2014; Sokol-Hessner et al, 2015), but in contrast to some prior findings that have shown that stress hormone actions, particularly cortisol, go along with sensitivity to risk (Coates and Herbert, 2008; Starcke and Brand, 2012; Kandasamy et al, 2014). However, while our task allowed disentangling loss from risk aversion and choice consistency (by using a prospect theory-driven adaptive design that maximized the information gained from each decision; Chumbley et al, 2014), other studies did not conceptually discriminate between these parameters with the same theoretical rigor. Our results replicate those of Chumbley et al (2014) and Sokol-Hessner et al (2015), who used the same task and structural model, and also found no relationship between endocrine stress markers and risk attitude, or choice consistency. These results suggest that stress-related neuromodulatory action might not have a general effect on risk attitude, but only on risky choices in which losses and gains are involved.

One limitation of our study is that we included only male participants in order to avoid an influence of hormonal variations due to the use of oral contraceptives or menstrual cycle phase that have been shown to affect cognition (Mather and Lighthall, 2012). There is evidence that males and females behave differently in economic decision making under stress (Croson and Gneezy, 2009). For example, women have been shown to become more risk averse and men more risk seeking under stress (Lighthall et al, 2009), and stress impacts reward-related decision processes differently for the two genders (Lighthall et al, 2012). Women have also shown to be more loss averse than men in certain situations (Rau, 2014). Future research should therefore investigate whether the results reported here generalize across genders.

In summary, we independently manipulated CORT and NA activity to disentangle their isolated and combined causal effects on decision-making. This is a crucial feature of our design because the two neuromodulators in combination affect cognition differently than they do in isolation (Joels and Baram, 2009; van Stegeren et al, 2010; Joëls et al, 2011; Schwabe et al, 2012; Vinkers et al, 2013). Our main finding, that combined action of CORT and NA diminished loss aversion relative to the action of either neuromodulator alone substantiates the body of research, and extends these findings to decision-making under risk. Further, our behavioral task allows disentangling loss aversion from risk attitude and choice consistency, while maximizing the informational gain from each participants' decision, and thus reducing measurement noise. An interesting expansion of the current findings would be to investigate the underlying neural activation during the decision making tasks using fMRI.

Our findings highlight the effect of combined NA and CORT action on loss aversion, and thus provide further insight into how acute stress, associated with concurrent NA and CORT activity, may lead to poor decision making. Increased hypersensitivity to reward paired with reduced sensitivity to punishment may result in heightened susceptibility to substance abuse (Lovallo, 2008). Thus, our findings have particular relevance to vulnerable populations such as drug users, problem gamblers, and other individuals suffering from addiction.

\section{FUNDING AND DISCLOSURE}

AS has been serving as a consultant for Medtronic Inc, Boston Scientific, St. Jude Medical, Grünenthal and has received lecture fees from Abbvie, Boston Scientific, St. Jude Medical, Medtronic Inc, UCB, MEDA Pharma, Teva Pharma, and GlaxoSmithKline. AS is a government employee and receives through his institution funding for his research from the German Research Council, the German Ministry of Education and Health, and the Gossweiler Foundation. TK's and ZM's work has been funded by the Deutsche Forschungsgemeinschaft (German Research Council, DFG-KA 2675/4-1 to TK). MVN received funding from the Volkswagen Stiftung 'Freigeist' initiative, AZ: 88216. LS and GN have no funding sources to declare. 


\section{ACKNOWLEDGMENTS}

The study was supported by a grant from the Deutsche Forschungsgemeinschaft to TK (DFG-KA 2675/4-1).

\section{REFERENCES}

Bos R van den, Harteveld M, Stoop H (2009). Stress and decisionmaking in humans: performance is related to cortisol reactivity, albeit differently in men and women. Psychoneuroendocrinology 34: 1449-1458.

Canessa N, Crespi C, Motterlini M, Baud-Bovy G, Chierchia G, Pantaeo G et al (2013). The functional and structural neural basis of individual differences in loss aversion. J Neurosci 33: 14307-14317.

Carver CS, White TL (1994). Behavioral inhibition, behavioral activation, and affective responses to impending reward and punishment: The BIS/BAS scales. J Pers Soc Psychol 67: 319-333.

Chumbley JR, Krajbich I, Engelmann JB, Russell E, Uum S Van, Koren $\mathrm{G}$ et al (2014). Endogenous cortisol predicts decreased loss aversion in young men. Psychol Sci 25: 2102-2105.

Coates JM, Herbert J (2008). Endogenous steroids and financial risk taking on a London trading floor. Proc Natl Acad Sci USA 105: 6167-6172.

Croson R, Gneezy U (2009). Gender differences in preferences. $J$ Econ Lit 47: 448-474.

Davis MH (1980). A multidimensional approach to individual differences in empathy. JSAS Cat Sel Doc Psychol 10: 85.

Elman I, Becerra L, Tschibelu E, Yamamoto R, George E, Borsook D (2012). Yohimbine-induced amygdala activation in pathological gamblers: a pilot study. PLoS ONE 7: e31118.

Gelskov SV, Henningson S, Madsen KH, Siebner HR, Ramsoy TZ (2015). Amygdala signals subjective appetitiveness and aversiveness of mixed gambles. Cortex 66: 81-90.

Gullo MJ, Stieger AA (2011). Anticipatory stress restores decisionmaking deficits in heavy drinkers by increasing sensitivity to losses. Drug Alcohol Depend 117: 204-210.

Hermans EJ, Henckens MJ, Joels M, Fernandez G (2014). Dynamic adaptation of large-scale brain networks in response to acute stressors. Trends Neurosci 37: 304-314.

Hermans EJ, Marie HJ van, Ossewarde L, Heckens MJ, Qin S, van Kesteren MT et al (2011). Stress-related noradrenergic activity prompts large-scale neural network reconfiguration. Science (80-) 334: 1151-1153.

Joels M, Baram TZ (2009). The neuro-symphony of stress. Nat Rev Neurosci 10: 459-466.

Joëls M, Fernandez G, Roozendaal B (2011). Stress and emotional memory: a matter of timing. Trends Cogn Sci 15: 280-288.

Kahneman D, Tversky A (1979). Prospect theory: an analysis of decision under risk. Econometrica 47: 263-292.

Kandasamy N, Hardy B, Page L, Schaffner M, Graggaber J, Powlson AS et al (2014). Cortisol shifts financial risk preferences. Proc Natl Acad Sci USA 111: 3608-3613.

Kinner VL, Wolf OT, Merz CJ (2016). Cortisol alters reward processing in the human brain. Hormones Behav 84: 75-83.

Kirschbaum C, Kudielka BM, Gaab J, Schommer NC, Hellhammer DH (1999). Impact of gender, menstrual cycle phase, and oral contraceptives on the activity of the hypothalamus-pituitary-adrenal axis. Psychosom Med 61: 154-162.

Lovallo W (2008). Cortisol secretion patterns in addiction and addiction risk. Int J Psychophysiol 59: 195-202.

Lighthall NR, Mather M, Gorlick MA (2009). Acute stress increases sex differences in risk seeking in the balloon analogue risk task. PloS ONE 4: e6002.

Lighthall NR, Sakaki M, Vasunilashorn S, Nga L, Somayajula S, Chen EY et al (2012). Gender differences in rewardrelated

decision processing under stress. Soc Cogn Affect Neurosci 7: 476-484.

Lupien SJ, Gillin CJ, Hauger RL (1999). Working memory is more sensitive than declarative memory to the acute effects of corticosteroids: a dose-response study in humans. Behav Neurosci 113: $420-443$.

Margittai Z, Nave G, Stromach T, Wingerden M, van, Schwabe L, Kalenscher T (2016). Exogenous cortisol causes a shift from deliberative to intuitive thinking. Psychoneuroendocrinology 64: 131-135.

Marinelli M, Piazza PV (2002). Interaction between glucocorticoid hormones, stress and psychostimulant drugs. Eur J Neurosci 16: 387-394.

Markett S, Heeren G, Montag C, Weber B, Reuter M (2016). Loss aversion is associated with bilateral insula volume. A voxel based morphometry study. Neurosci Lett 619: 172-176.

De Martino B, Camerer CF, Adolphs R (2010). Amygdala damage eliminates monetary loss aversion. Proc Natl Acad Sci USA 107: 3788-3792.

Mather M, Lighthall NR (2012). Both risk and reward are processed differently in decisions made under stress. Curr Dir Psychol Sci 21: $36-41$.

Meule A, Vögele C, Kübler A (2011). Psychometrische evaluation der deutschen Barratt impulsiveness scale - Kurzversion (BIS-15). Diagnostica 57: 126-133.

Montoya ER, Bos PA, Terburg D, Rosenberger LA, van Honk J (2014). Cortisol administration induces global down-regulation of the brain's reward circuitry. Psychoneuroendocrinology 47: 31-42.

Oei NYL, Both S, van Heemst D, van der Grond J (2014). Acute stress-induced cortisol elevations mediate reward system activity during subconscious processing of sexual stimuli. Psychoneuroendocrinology 39: 111-120.

Pabst S, Brand M, Wolf OT (2013a). Stress effects on framed decisions: there are differences for gains and losses. Front Behav Neurosci 7: 142.

Pabst S, Brand M, Wolf OT (2013b). Stress and decision making: a few minutes make all the difference. Behav Brain Res 250: 39-45.

Paulus C (2007). Der Saarbrückener Persönlicheitsfragebogen SPF (IRI) http://psydok.sulb.uni-saarland.de/volltexte/2009/2363/.

Piazza PV, Moal ML (1997). Glucocorticoids as a biological substrate of reward: physiological and pathophysiological implications. Brain Res Rev 25: 359-372.

Porcelli AJ, Delgado MR (2009). Acute stress modulates risk taking in financial decision making. Psychol Sci 20: 278-283.

Porcelli AJ, Delgado MR (2017). Stress and decision making: effects on valuation, learning and risk taking. Curr Opin Behav Sci 14: 33-39.

Pruessner JC, Kirschbaum C, Meinlschmid G, Hellhammer DH (2003). Two formulas for computation of the area under the curve represent measures of total hormone concentration versus timedependent change. Psychoneuroendocrinology 28: 916-931.

Putman P, Antypa N, Crysovergi P, Van Der Does WaJ (2010). Exogenous cortisol acutely influences motivated decision making in healthy young men. Psychopharmacology 208: 257-263.

Randler C (2013). German version of the reduced MorningnessEveningness Questionnaire (rMEQ). Biol Rhythm Res 44: 730-736.

Rau HA (2014). The disposition effect and loss aversion: Do gender differences matter? Econ Lett 123: 33-36.

Rogers RD, Lancaster M, Wakeley J, Bhagwagar Z (2004). Effects of beta-adrenoceptor blockade on components of human decisionmaking. Psychopharmacology 172: 157-164.

Rohleder N, Nater UM, Wolf JM, Ehlert U, Kirschbaum C (2004). Psychosocial stress-induced activation of salivary alpha-amylase: an indicator of sympathetic activity? Ann NY Acad Sci 1032: 258-263.

Schwabe L, Tegenthoff M, Höffken O, Wolf OT (2010). Concurrent glucocorticoid and noradrenergic activity shifts instrumental 
behavior from goal-directed to habitual control. J Neurosci 30: 8190-8196.

Schwabe L, Tegenthoff M, Höffken O, Wolf OT (2012). Simultaneous glucocorticoid and noradrenergic activity disrupts the neural basis of goal-directed action in the human brain. J Neurosci 32: $10146-10155$.

Sinha R (2008). Chronic stress, drug use and vulnerability to addiction. Ann NY Acad Sci 1141: 105-130.

Sokol-Hessner P, Camerer CF, Phelps EA (2013). Emotion regulation reduces loss aversion and decreases amygdala responses to losses. Soc Cogn Affect Neurosci 8: 341-350.

Sokol-Hessner P, Hsu M, Curley NG, Delgado MR, Camerer CF, Phelps EA (2009). Thinking like a trader selectively reduces individuals' loss aversion. Proc Natl Acad Sci USA 106: 5035-5040.

Sokol-Hessner P, Lackovic SF, Tobe RH, Camerer CF, Leventhal BF, Phelps EA (2015). Determinants of propranolol's selective effect on loss aversion. Psychol Sci 26: 1123-1130.

Sokol-Hessner P, Raio CM, Gottesman SP, Lackovic SF, Phelps EA (2016). Acute stress does not affect risky monetary decisionmaking. Neurobiol Stress 5: 19-25.

Spielberger CD, Gorsuch RL, Lushene R, Vagg PR, Jacobs GA (1983). Manual for the State-Trait Anxiety Inventory. Consulting Psychology Press: Palo Alto, CA, USA.

Starcke K, Brand M (2012). Decision making under stress: a selective review. Neurosci Biobehav Rev 36: 1228-1248.
Starcke K, Brand M (2016). Effects of stress on decisions under uncertainty: a meta-analysis. Psychol Bull 142: 909-933.

Stegeren AH, van, Roozendaal B, Kindt M, Wolf OT, Joëls M (2010). Interacting noradrenergic and corticosteroid systems shift human brain activation patterns during encoding. Neurobiol Learn Mem 93: 56-65.

Stegeren AH, van, Wolf OT, Everaerd W, Rombouts SA (2008). Interaction of endogenous cortisol and noradrenaline in the human amygdala. Progress Brain Res 167: 263-268.

Ströber J (2001). The Social Desirability Scale-17 (SDS-17): convergent validity, discriminant validity, and relationship with age. Eur J Psychol Assess 17: 222-232.

Takahashi H, Fujie S, Camerer C, Arakawa R, Takano H, Kodaka F et al (2012). Norepinephrine in the brain is associated with aversion to financial loss. Mol Psychiatry 18: 3-4.

Vasa Ra, Pine DS, Masten CL, Vythilingam M, Collin C, Charney DS et al (2009). Effects of yohimbine and hydrocortisone on panic symptoms, autonomic responses, and attention to threat in healthy adults. Psychopharmacology 204: 445-455.

Vinkers CH, Zorn JV, Cornelisse S, Koot S, Houtepen LC, Olivier B et al (2013). Time-dependent changes in altruistic punishment following stress. Psychoneuroendocrinology 38: 1467-1475.

Wang SW, Filiba M, Camerer CF (2010). Dynamically Optimized Sequential Experimentation (DOSE) for Estimating Economic Preference. 1-41. 